\title{
The use of the static thermography in monitoring flap perfusion in breast reconstruction with TRAM flap
}

\author{
by Sz. Kołacz*, M. Moderhak** , J. Jankau* \\ * Department of Plastic Surgery, Medical University of Gdańsk, ul. Debinki 7, 80-211, Gdańsk, \\ skolacz@gmail.com \\ ** Department of Biomedical Engineering, Gdańsk University of Technology, 80-233, ul. \\ Narutowicza, Gdańsk, Poland, matmod@biomed.eti.pg.gda.pl
}

Acknowledgement: This work has been partially supported by Statutory Funds of Electronics, Telecommunications and Informatics Faculty, Gdansk University of Technology

\begin{abstract}
This paper shows results of the static thermography for intraoperative and postoperative imaging of TRAM flap perfusion. The results were compared with the clinical examination of flap perfusion. The study was conducted on a group of 38 female patients who underwent breast reconstruction.
\end{abstract}

\section{Introduction}

One of the primary tasks of plastic surgery is the reconstruction of integumentary defects with the use skinfat or skin-muscle flaps. The flaps can be both pedicled and free, in which the flap vessels are connected with the recipient site vessels with microsurgical techniques. They involve surgical procedures with a high risk of complications, ranging from partial marginal necrosis with total necrosis of the flap. The consequences include a long hospitalization, increased risk of surgical site infection, necessity for subsequent operations, increased costs of treatment, and - finally - a poorer aesthetic and functional result.

The literature on the subject presents a number of methods for monitoring blood supply to the flap. They allow for more accurate and faster evaluation of perfusion impairment, which, in turn, could contribute to earlier surgical intervention and consequently reduce the adverse effects of ischemia. Methods used in evaluating blood supply to the flap include: MRI, CT, Doppler laser, Doppler ultrasound, angiography and thermography [1,2]. Not all of them, however, meet the conditions for an ideal method, such as a lack of invasiveness, reproducibility of results, intraoperative or bedside procedures, ease of use and low cost of purchase and operation. Thermography is the closest to meeting those criteria.

\section{Problems}

TRAM flap is among the most frequently applied pedicled skin-muscle flaps in reconstructive procedures. It is based on the superior epigastric artery. Despite the well-researched anatomy of the TRAM flap, complications may develop manifesting as partial flap necrosis due to impaired blood supply. Hartrampf described four zones of the flap blood supply [3,4]. Zones I and II receive the best blood supply, which is why they are applied in reconstructive procedures. Zones III and IV are resected due to poor vasculature leading to necrosis. The division into zones is indicative rather than precise. The surgeon's experience is very important in defining the zones. Adequate resection of zones III and IV helps avoid potential complications, but it should be noted that resecting excess tissue with segments of zones I and II may result in tissue volume which is insufficient for breast reconstruction or may lead to other integumentary loss. Consequently, what is crucial is both the surgeon's experience and assistance in the form of various imaging methods, while the application of static thermography may contribute to more precise assessment of flap perfusion. 


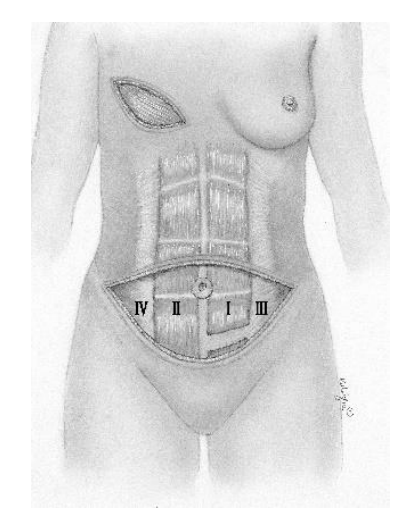

Fig. 1. TRAM flap zones

There is available literature on the use of static thermography in postoperative monitoring of vascular perfusion in a free TRAM flap in which temperature decrease within a short period indicates lack of patency in the microsurgical anastomosis [5]. Together with changes revealed in a clinical examination, it is an indication for instant surgical intervention. Preoperative mapping of vascular perforators used in microsurgical anastomoses is another kind of described applications of static thermography [6]. There are, however, no available unequivocal descriptions of static thermography application in the intraoperative and postoperative assessment of pedicled flap blood supply in humans with a simultaneous comparison of the clinical assessment.

The aim of this paper was to compare the intraoperative and postoperative clinical assessment of flap perfusion with temperature value measured in static thermography in breast reconstructive procedure with the use of pedicled TRAM flaps, noting the fact that the relationship between skin temperature and blood supply is welldocumented in literature [7].

\section{Method and results}

This paper presents the results of a study group of 38 patients undergoing breast reconstruction with a pedicled skin-muscle TRAM flap, including 10 patients who underwent TRAM IPSI procedure, 10 - TRAM CONTRA and 18 - TRAM supercharged, in which inferior epigastric vessels were additionally anastomosed with internal thoracic vessels. Each patient was examined intraoperatively after the flap was dissected (b2), directly after the procedure was completed when the patient was still in the operating theater (b3), on Day 1 (b4) and Day 7 (b5) after the procedure. The evaluation included static thermography examination and the clinical assessment of blood supply to the flap documented with a photographic examination. All surgical procedures were performed by the same surgical team and each patient had the opportunity to withdraw from the study at each stage. The study was approved by the Bioethic Committee.

The average age in the study group was 51.9 years (range of 30-67 years). The mean BMI was 28.7 (range of 21-41). In the studied group, the number of smokers in the last 90 days was 7 (18.4\%). 20 (52.6\%) patients had undergone chemotherapy, $10(26.3 \%)$ - radiotherapy. The period between the end of radiotherapy or chemotherapy and the surgery was 9-41 months.

All patients received general intratracheal anesthesia. The procedures were performed in the same constant temperature.

The operation began with skin incision and umbilicus dissection. Next the skin was incised along the entire length of the skin-fat flap to the right anterior superior iliac spine from the left one. The superior line delimiting the flap was the superior edge of the umbilicus and the lower distance of $7 \mathrm{~cm}$ from the superior angle of the labial commissure.

Then a dissection was directed from the side medially on the surface of muscle fascia until we reached the lateral edge of the rectus abdominal muscle. An incision was made on the anterior wall of the sheath to uncover inferior epigastric vessels, which were dissected, ligated and then cut along with a simple abdominal muscle. Subsequently, a skin-fat flap was lifted from abdominal integuments, with the upward direction of the dissection, up 


\subsection{1/qirt.2016.060}

to the border of the costal arch and xiphoid. Along the entire length of the rectus abdominis muscle, the anterior sheath was incised, leaving a $3 \mathrm{~cm}$ wide strip of fascia in order to protect against the excessive extension of the pedicle and to enable lifting the skin-muscle TRAM flap. Throughout the operation hemostasis was carefully managed: the accompanying blood vessels were coagulated or ligated. At this stage the TRAM flap was appropriately modelled by means of dissecting the entire zone IV, with a part of zone II and III.

The recipient region was prepared by incising the amputation cicatrix, lifting the dermal-subcutaneous flaps and dissecting a subcutaneous tunnel in the region of the xiphoid and costal arch. Then the flap was transferred into the reconstructed area to form a new breast mound.

In TRAM Supercharged flap operations, after exposing the lodge at the recipient site, a segment of rib 4 cartilage was also dissected in order to obtain access to the internal thoracic vessels. We used microscopic monitoring - Carl Zeiss OPMI Vario / S88, magnification of 1:6 - while dissecting the internal thoracic vessels which were anastomosed with inferior epigastric vessels with a Prolene 8-0 suture visualizing the arterial and venous inflow, following the flap transfer.

Following the abdominal flap incision, the umbilicus was brought out and sutured with Vicryl 4-0 and 5-0 Nylon. The wounds were closed with Vicryl 2-0 and 4-0 sutures, and V-Loc 4-0 wound closure device, respectively.

Each operated patient underwent a thermographic examination performed in compliance with the same standards with FLIR A320G camera with spatial resolution of $320 \times 240$ pixels and thermal resolution of $0.08 \mathrm{~K}$. The camera was positioned at a distance of $70 \mathrm{~cm}$ from the studied flap and perpendicularly to its maximum mound. The first imaging examination was performed immediately after the dissection of the skin-muscle flap and excision of its excesses, second after suturing flap in the recipient region, and the follow-up ones - at 1 and 7 days after the surgery. Each examination consisted of the following steps:

- static thermal imaging

- clinical examination of flap

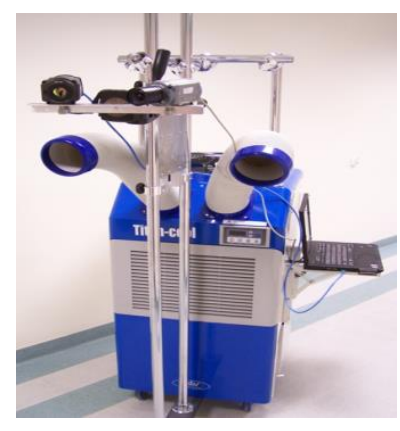

Fig. 2. Thermographic Station

The collected data were then processed to yield results in a numerical form. In the case of static thermography, we assessed the average temperature $T_{\text {stat }}$ of the flap, expressed in Kelvin degrees.

In order to increase the accuracy of the imaging of skin blood supply disorders in the static tests, each flap was divided into eight zones: 4 central zones $(C 1, C 2, C 3, C 4)$ and 4 peripheral zones (B1, B2, B3, B4). The division was always proportional to the size of the examined area. 


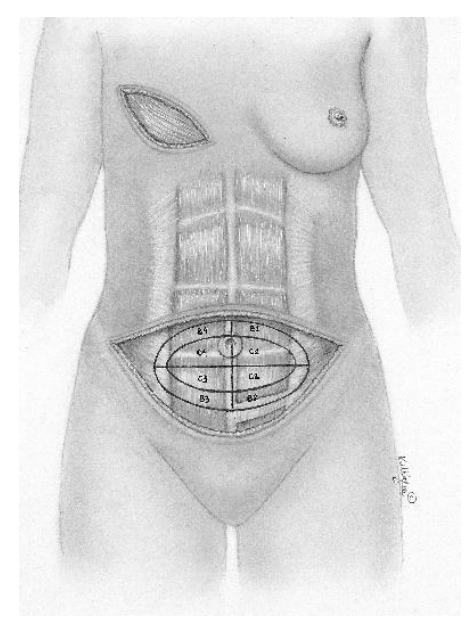

Fig. 3. Flap scheme before rotation

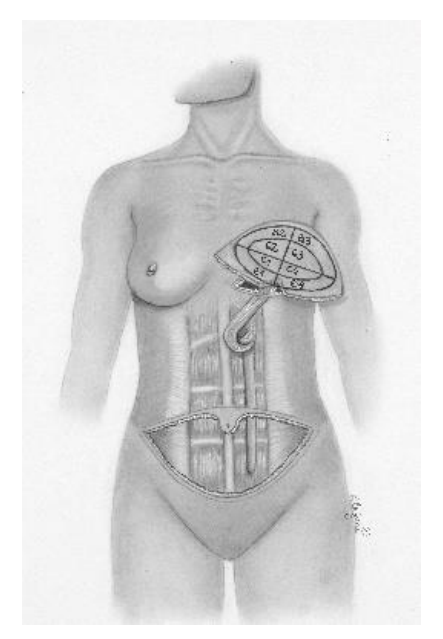

Fig. 4. TRAM IPSI after rotation

As a result, each piece could be clearly identified despite the change in the spatial arrangement of the flap during reconstructive surgery. For each zone both $\mathrm{T}_{\text {stat }}$ values were calculated. The obtained data were analyzed statistically and compared with the clinical examination results.

Before the initiation of the surgery, the presence of perforators at hot spots of the thermographic examination was confirmed with a Doppler ultrasound probe.

Throughout the period of research photographic documentation was maintained. Throughout the research program no tissue infection or hematomas developed at the recipient site.

All statistical calculations were performed with the use of a statistical package - StatSoft. Inc. (2011). STATISTICA (data analysis software system) version 10.0 www.statsoft.com and an Excel spreadsheet. Quantitative variables were presented with the use of the arithmetic mean, standard deviation, median, minimum and maximum values (range) and $95 \% \mathrm{Cl}$ (confidence interval), while qualitative variables were presented with the use of frequencies and percentage values. In order to verify whether a quantitative variable came from a normally distributed population the Shapiro-Wilk test was used. The Leven (Brown-Forsythe) test was used to the hypothesis of equal variances.

The significance of differences between the two groups (unpaired variables) was examined with significance difference tests: the Student's $t$ (or, in the absence of homogeneity of variance - Welch test) or MannWhitney $U$ test (where the conditions of applicability are not met for Student's t-test or for variables measured on the ordinal scale). The significance of differences between more than two groups was tested with an $F$ test (ANOVA) or Kruskal-Wallis test (where the conditions of applicability are not met for ANOVA). If statistically significant 


\subsection{1/qirt.2016.060}

differences were found between groups, post hoc tests were applied (Tukey test for F. Dunn - for the Kruskal-Wallis test). In the case of two paired variables Student's t test or Wilcoxon matched pairs test was used (if applicability conditions were not met for the Student t-test or for variables measured on the ordinal scale). The significance of differences between more than two related variables in the model analysis of variance was tested with repeated measurements or Friedman's test (in the case of non-compliance with the applicability of the analysis of variance with repeated measures or for variables measured on an ordinal scale). Chi-square independence tests were used for categorical variables (applying correction by Yates for cell number smaller than 10, verification of Cochran conditions, Fisher's exact test, respectively).

In all the calculations the level of significance was set at $p=0.05$.

\section{Results}

The table below presents the results of a comparative analysis of static thermography performed after flap dissection (b2), immediately after flap rotation and suturing and postoperatively (b3): on Day 1 (b4) and 7 (b5) after surgery. Out of 38 patients participating in the study, 9 patients developed marginal necrosis of the skin flap despite intraoperative clinical evaluation of blood supply. No flap developed total necrosis, which would have indicated pedicle tightening or cutting. In the case of TRAM supercharged it would have additionally indicated microsurgical anastomosis obstruction.

Table 1. Comparison of the mean values from necrotic quadrants of all flaps in static thermography as per complication incidence in test b-2, b-3, b-4 and b-5.

\begin{tabular}{|c|c|c|c|}
\hline & $\begin{array}{l}\text { without complications } \\
\qquad \mathrm{n}=29\end{array}$ & $\begin{array}{c}\text { complications } \\
\mathrm{n}=9\end{array}$ & $p$ value \\
\hline \multicolumn{4}{|l|}{ b-2 } \\
\hline mean (SD) & $301,64(1,43)$ & $301,69(1,42)$ & \\
\hline $95 \% \mathrm{Cl}$ & {$[301,47 ; 301,82]$} & {$[301,01 ; 302,37]$} & \\
\hline range (min-max) & $298,36-304,95$ & $299,93-303,81$ & \\
\hline Median & 301,50 & 301,34 & 0,9341 \\
\hline \multicolumn{4}{|l|}{ b-3 } \\
\hline mean (SD) & $301,75(1,88)$ & $301,79(2,04)$ & \\
\hline $95 \% \mathrm{Cl}$ & {$[301,52 ; 301,97]$} & {$[300,80 ; 302,77]$} & \\
\hline range (min-max) & $297,68-307,33$ & $299,15-306,44$ & \\
\hline Median & 301,53 & 301,83 & 0,9409 \\
\hline \multicolumn{4}{|l|}{ b-4 } \\
\hline mean (SD) & $307,23(1,51)$ & $305,27(1,45)$ & \\
\hline $95 \% \mathrm{Cl}$ & {$[307,05 ; 307,41]$} & {$[304,55 ; 305,99]$} & \\
\hline range (min-max) & $302,86-309,90$ & $303,31-308,12$ & \\
\hline Median & 307,49 & 305,08 & 0,0001 \\
\hline \multicolumn{4}{|l|}{ b-5 } \\
\hline mean (SD) & $307,20(1,49)$ & $306,10(1,51)$ & \\
\hline $95 \% \mathrm{Cl}$ & {$[307,03 ; 307,38]$} & {$[305,14 ; 307,06]$} & \\
\hline range (min-max) & $302,71-311,21$ & $303,41-308,37$ & \\
\hline Median & 307,32 & 306,07 & 0,0165 \\
\hline
\end{tabular}

Table 1 presents static thermography results. Mean values of necrotic quadrants from all flaps were compared to the mean value of all non-necrotic flaps with respect to examination period. Both in the intraoperative test and in the test performer directly after the flap was sutured onto the recipient site, the standard deviation (SD), the median and the mean value of the temperature did not show statistically significant differences between the flaps with and without complications. After 24 hours, however, the difference of all three parameters was significant, 


\subsection{1/qirt.2016.060}

which was also confirmed in a thermographic examination on Day 7. Signs of impaired flap perfusion such as skin color, capillary refill time or flap temperature were visible in a simultaneous clinical evaluation as late as after 24 hours and persisted until Day 7, when necrotic tissue was removed. The illustrations below show images of Patient 6 and static thermograms.
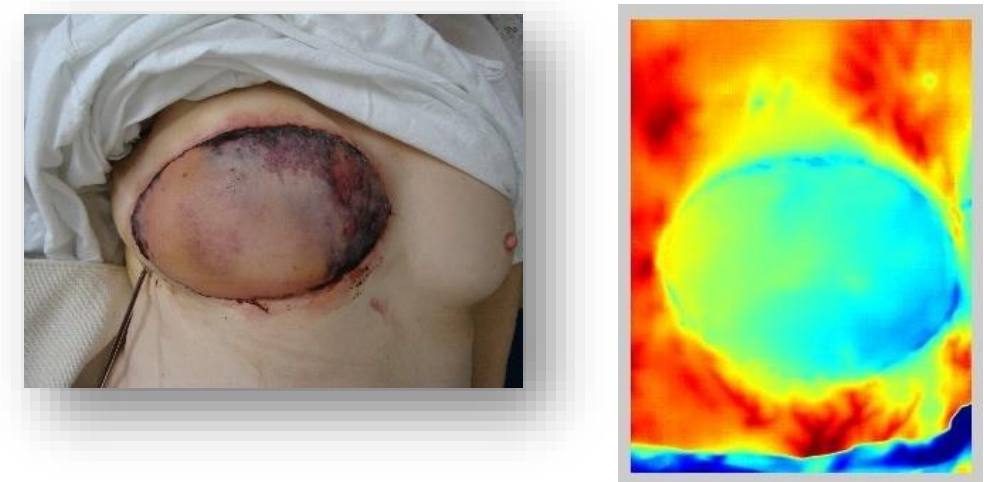

Fig. 5. Partial TRAM flap necrosis. Day 7 after surgical procedure
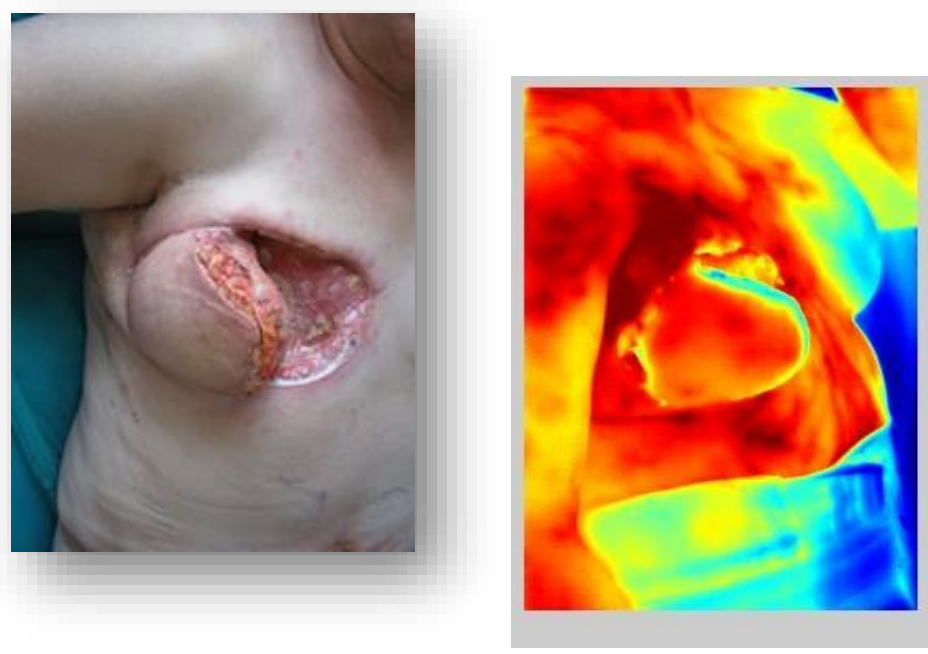

Fig. 6. TRAM flap after debridement. Day 21 after surgical procedure

There is no available literature describing the application of static thermography in humans to evaluate pedicled flap perfusion intraoperatively and on subsequent postoperative days, and therefore literature discussion is not possible. Following the analysis of the presented results we think that static thermography does not add significant value to intraoperative evaluation of potential necrosis. It is, however, thoroughly recommended as an imaging examination performed within 24 hours after the procedure and on subsequent postoperative days to support and provide adequate documentation of clinical assessment.

\section{REFERENCES}

[1] Barry M. Jones. "Monitors for the Cutaneous Microcirculation" Plastic and Reconstructive Surgery, May 1984

[2] Creech, B., and Miller, S. "Evaluation of Circulation in Skin Flaps". In W. C. Grabb and M. B. Myers (Eds.), Skin Flaps. Boston: Little, Brown, 1975

[3] Hartrampf CRJr, Scheflan M, Black PW. "Breast reconstruction with a transverse abdominal island flap". Plast Reconstr Surg. 69;216-225, 1982. 


\subsection{1/qirt.2016.060}

[4] Moon HK, Taylor GI. "The Vascular Anatomy of Rectus Abdominis Musculocutaneous Flaps Based on the Deep Superior Epigastric System”. Plast Reconstr Surg Vol. 82, No. 51998.

[5] Roger K. Khouri, William W. Shaw. "Monitoring of Free Flaps with Surface-Temperature Recordings: Is It Reliable?" Plastic and Reconstructive Surgery, March 1992

[6] A.M. Salmi, E. Tukiainen, S. Asko-Seljavaara. Thermographic Mapping of Perforators and Skin Blood Flow in the Free Transverse Rectus Abdominis Musculocutaneous Flap. Annals of Plastic Surgery Vol. 35 Number 2 August 1995

[7] Francis JE, Roggli RI, Love TJ. "Thermography as means of blood perfusion measurement”. J Biomech Eng; $101 ; 246-251,1979$ 